\title{
Unusual Vascular Events in the Territory of the Posterior Cerebral Artery
}

\author{
C.M. Fisher
}

\begin{abstract}
There is an unusual type of vascular episode in the territory of the posterior cerebral artery which remains relatively unknown. Ten cases are presented in which a posterior cerebral artery deficit developed suddenly in dramatic fashion with headache, visual symptoms, sensory and motor deficits and signs of third nerve involvement. Nine of the patients were female and one was male. Seven were under the age of 33 . In all instances there was a permanent neurologic sequela, usually a hemianopia. A similar case was described in 1901. The nature of the underlying process remains obscure, but the evidence favors accompanied migraine in which a particularly severe attack results in permanent damage. The term "catastrophic migraine" is suggested.
\end{abstract}

RÉSUMÉ: Evénements vasculaires inhabituels dans le territoire de l'artère cérébrale postérieure ll existe une forme relativement inconnue d'épisode vasculaire qui se produit dans le territoire de l'artère cérébrale postérieure. Nous discutons de 10 cas chez qui un déficit dans le fonctionnement de l'artère cérébrale postérieure s'est manifesté soudainement et de façon dramatique, par une céphalée, des symptômes visuels, des déficits sensorimoteurs et des signes d'atteinte du 3e nerf crânien. Il y avait 9 femmes et un homme dans ce groupe. Sept de ces patients étaient âgés de moins de 33 ans. Dans tous les cas on note la persistance d'une séquelle neurologique, surtout d'une hémianopie. Un cas semblable a été décrit en 1901. La nature du processus sous-jacent est encore inconnue, mais l'évidence favorise la présence d'une migraine accompagnée d'une intensité telle qu'il subsiste une lésions permanente. Nous suggérons le terme "migraine catastrophique" pour décrire ce phénomène.

Can. J. Neurol. Sci. 1986: 13:1-7

Most stroke events involving the posterior cerebral artery (PCA) territory, whether embolic, thrombotic or migrainous, follow well-recognized patterns. There are some cases, however, in which the clinical features are so unusual and the nature of the process so uncertain that, for the most part, they have gone unreported in the literature. Ten such cases have been selected for presentation here. In these patients a PCA deficit has developed in dramatic fashion to the accompaniment of headache, visual symptoms, sensory and motor deficits, and sometimes signs of third nerve involvement. The patients have usually been female and much younger than the ordinary stroke age. In all instances there has been a prolonged or permanent neurologic sequela. The cases are both rare and puzzling with the result that it has taken a considerable time to recognize that they have enough features in common to represent a clinical syndrome.

\section{Case Histories}

Although they share many features, the cases are in other ways distinctive and varied; and to provide an adequate view of the spectrum, it is necessary to present a description of each case.
Case 1: A man aged 30 awakened at 3 a.m. with a very severe bitemporal pounding headache. He fell asleep again and on getting up at $6 \mathrm{a} . \mathrm{m}$. dropped things from his left hand. On investigating the reason for this he found that the left arm and leg were totally without feeling. When examined at a local first-aid station, he did not feel pinprick. If he looked at his fingers, he could move them with full power. There was almost full restoration of feeling in three hours, but the fingertips remained slightly numb for several weeks. The patient disclaimed ever having had a headache in the past, but afterwards moderately severe headaches recurred regularly.

Five months later on Christmas morning he awakened and went to the Christmas tree normally. Almost immediately he became dizzy, sat down, put his head between his knees, and said he could not see anything. He answered a few questions clearly, but speech then became slurred. He made dysphasic errors and in a minute or two became virtually mute. At the same time, his right arm and leg became weak and eventually totally paralyzed. About 25 minutes from the onset he began to move his right arm again, slurred speech reappeared and his wife noted that his right eye was turned far laterally and could not be brought to the midline. The left eye remained in mid position. He noted diplopia. Thirty minutes from the onset he developed a severe headache. He was treated with steroids intravenously and by 1530 , seven hours from the onset, he talked and walked normally.

There was sharp pain in the left side of the head and face. In the evening a right hemiparesis and dysarthria returned and he used word substitutions. On day 2 a dense right homonymous hemianopia, reading difficulty and a severe sensory loss on the right side were noted. His

This paper was presented as part of the J.L. Silversides Lecture at the Academy of Medicine. Toronto, June 1985.

From the Neurology Service of the Massachusetts General Hospital, Boston, Massachusetts 02114

Reprint requests to: C. Miller Fisher, M.D., Neurology Service, Massachusetts General Hospital, Fruit Street, Boston, Mass., U.S.A. 02114 
neurological deficits fluctuated in the following week at the end of which time his right hand was still weak and clumsy and speech was slurred and dysphasic errors occurred. The right field defect and dyslexia continued. The headache subsided in four days.

Gradual improvement occurred and examination in the hospital five weeks later showed a right homonymous hemianopia denser in the upper quadrant. Reading the right hand side of words was slow and faulty. There was no defect in the use of proper names (relatives, towns, motor cars), in color naming or spelling. The patient's memory and intellect were judged to be normal except for a morbid preoccupation and obsession with his visual field defect. Speech was slightly slurred, the right hand was slightly clumsy, and there was a slight limp. There was a slight cerebellar ataxia on the right finger-nose test and a gross dysmetria on the finger-on-thumb tapping test. Sensation was intact except that pinprick felt sharper on the left side. The plantar responses were flexor.

While in the hospital, he had an episode of numbness beginning in the right index finger and spreading in 10 to 20 minutes to the other fingers up to the elbow and finally to the right cheek. Seven hours after his angiogram, he developed "wavy" cloudiness in the left visual field progressing for 30 minutes and suddenly disappearing.

A CT scan showed a large low density area in the territory of the left PCA (Figure 1). There was a $1 \mathrm{~cm}$ low density area in the left anterior thalamus. Vertebral-basilar digital subtraction angiography disclosed occlusion of the left PCA posterior to the thalamus at the origin of the posterior inferior temporal branch (Figure 2). Extensive laboratory investigations were otherwise negative.

Comment: It is likely that the territory of the right PCA was involved in the first episode, that of the left PCA in the second episode. In the second, the precommunal segment of the left PCA was involved and possibly both PCAs judging from the report of lateral deviation of the right eye. In spite of bilateral involvement, consciousness was not impaired. Headache was prominent in each episode. In the second episode, the neurologic deficit developed serially in the manner of migraine. Occlu- sion of the left PCA was too far distal to account for the mesencephalothalamic deficits. The nature of the process will be discussed later in the context of all the cases. The spreading paresthesias and the gradually progressive cloudiness of vision experienced in the hospital are typical of migraine accompaniments.

Case 2: A woman aged 27 with a history of accompanied migraine was informed that her brother, of whom she was particularly fond, had just been killed in an automobile accident. She was distraught and developed a sharp headache in the right forequarter of her head along with fuzziness of vision. Within minutes (immediately, according to one report) she developed a total left hemiplegia and inability to see to the left side. On arrival at the hospital, there was a dense left homonymous hemianopia. A severe paralysis of the left limbs proved to be largely due to a profound sensory loss to all modalities throughout the left side. When motor testing of the left limbs was carried out with the limbs visible to the patient, power approached normal in both the arm and the leg. Any weakness of the left side of the face was questionable, but the plantar response on the left was extensor and there was a right gaze preference. In the following four weeks the field defect sensory deficit and motor function returned almost to normal. Eight weeks from the onset a movement disorder developed consisting of a resting tremor and choreic activity involving the left hand.

A CT scan on day 14 showed gray matter enhancement in the occipital territory of each PCA. Angiography showed occlusion of the right PCA far distally at approximately the origin of the parieto-occipital branch. There was transborderzone collateral flow. from the middle cerebral artery. An echocardiogram indicated a slight late systolic prolapse of the mitral valve. Examination of the CSF was normal, as were other laboratory tests.

Three years before this episode, when the patient was using oral contraceptive medication, she had been subject to frequent left-sided headaches accompanied by nausea, vomiting and "moving lines" in her vision. There were occasional episodes of right-sided numbness and one in which the right side, face, arm and leg were weak with

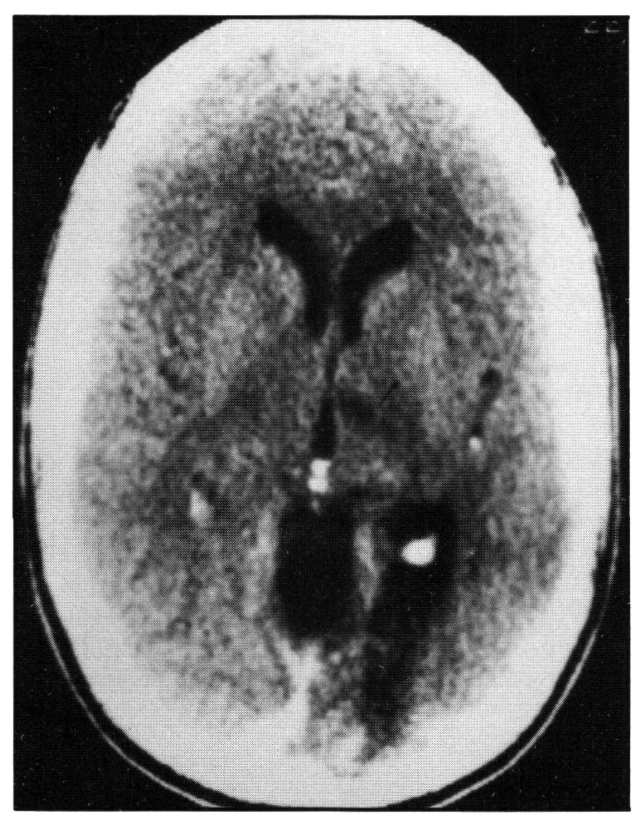

Figure I - CT Scan in Case I showing hypodense regions in left occiptal lobe and anterior thalamus.

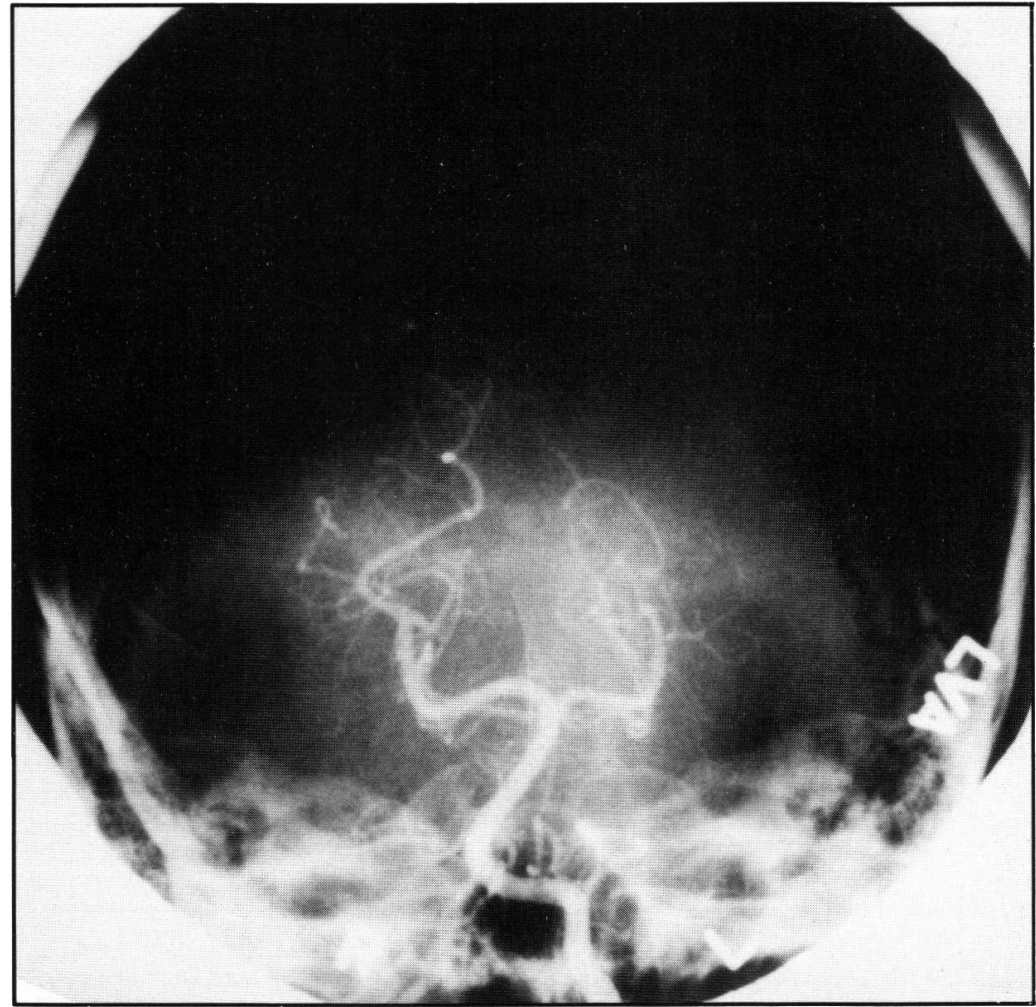

Figure 2 - Angiogram in Case 1 showing occulsion of left posterior cerebral artery. 
recovery taking five days. On stopping oral contraceptives these symptoms ceased.

Two years later, the movement disorder of the left hand was still present and the left side was hyperesthetic to stimulation.

Comment: The dramatic onset of symptoms at the time of an emotional shock is impressive. A profound sensory loss caused an apparent paralysis. Both occipital lobes showed enhancement on CT. The right PCA was occluded far distally. The possibility of embolism has to be considered. The post-stroke onset of choreiform activity is illustrated.

Case 3: A woman aged 32 subject to emotional strain developed a slight headache at the pate and an ache in the occipito-nuchal region. She retired, but in the next hour developed a "very, very bad" generalized headache associated with nausea and vomiting. While vomiting, the pain was so severe she cried out in alarm and in a flash felt "halfanesthetized". She was unable to move her limbs or open her eyes, she heard a low humming sound, her neck felt numb and the headache ceased abruptly. She kept her senses and was able to ask a servant to call an ambulance. She was totally blind and initially both eyelids were closed. When the lids were lifted, she could not see. She maintained awareness for the next eight hours, but had no memory for the following 24 hours. Twenty-four hours from the onset there was weakness of all four limbs and perioral numbness. The right eye opened, but the left lid remained ptosed. The left eye was displaced laterally and downwards and did not adduct. Vision began to return in 36 hours and after three days, she could see shapes, then colors and vision became normal in the right eye and $80 \%$ of normal in the left eye. The left arm remained weak for three weeks. A left inferior quadrantanopia persisted and diplopia was gradually diminishing when the patient was examined seven weeks from the onset.

At that time, memory and intellect were intact. The pupils were $2.5 \mathrm{~mm}$ in diameter and reactive. There was a left homonymous hemianopia much denser in the lower quadrant. Optokinetic nystagmus was symmetrical. The optic discs were normal. There was a $2 \mathrm{~mm}$ left ptosis. On forward gaze the left eye was displaced $3 \mathrm{~mm}$ laterally and $2 \mathrm{~mm}$ downwards and the patient noted diplopia. With special effort she saw singly. The remainder of the neurological examination was normal except for slightly increased tendon reflexes on the left side, slight unsteadiness of gait and a mild cerebellar dysmetria in the left arm.

A CT scan obtained 13 hours from the onset showed a small low density area in the right occipital lobe. A CT scan with contrast at 36 hours showed a large low density area in the territory of the right PCA and small areas of low density in the medial part of each thalamus. There was a questionable small area of low density in the mid portion of the pons.

Right vertebral and right carotid angiography showed patency of all arteries. However, the upper half of the basilar artery and both posterior cerebral arteries showed an unusual fine corrugation of the column of dye regarded by neuroradiological experts as definitely abnormal. The forward flow of blood was not impeded. An extensive series of laboratory tests were all normal.

There was no definite personal or family history of headache. Recent past history was notable in that the patient had undergone her third delivery by caesarean section three months before. Both the patient and her child had been well in the interim.

Comment: Again there was a cataclysmic onset marked by headache, blindness, ptosis, weakness of limbs and impaired memory. Both PCAs must have been involved as the hemianopia and third nerve palsy were on the same side. Although the PCAs were not obstructed, their walls and that of the basilar artery had an unusual irregularity.

Case 4: (This case was included as Case 57 in a previous paper: Fisher 1971.) A "nervous" girl aged 18, while arguing with her boyfriend, suddenly said, "I can't move," and almost immediately added, "I can't see." Some vision was restored in 30 minutes but she was unable to walk unaided. Speech was slurred, stuttering and "mixed up". In 24 hours the patient could walk with help and there was numbness of the right half of the lips, right side of the nose, ear, cheek, tongue, chin, neck and of the right arm and leg. Her right slipper came off without her being aware of it. There had been no headache. In five days walking was back to normal. Memory was impaired and the patient repeatedly asked the same questions. The numbness of the right arm and leg was almost gone after one month at which time a disabling tremor appeared in the right arm and fingers. Vision in the right field was impaired.

Examination one year after the acute incident disclosed a right homonymous hemianopia with greater impairment superiorly than inferiorly. There was a slight right facial weakness. When the arms were held extended, the fingers on the right and the proximal arm trembled rhythmically at about $3 / \mathrm{sec}$. This tremor became much greater on the finger-nose test. There was no tremor at rest. Sensation was intact although the patient complained of tingling of the right fingertips. The tendon reflexes were a little brisker on the left side. The patient recalled a ten point verbal story at 25 minutes. The blood pressure was $107 / 80 \mathrm{~mm} \mathrm{Hg}$.

The cerebrospinal fluid was normal. A technetium brain scan was normal. Right carotid and right brachial ateriograms showed no abnormality. There was no obvious source of embolism.

The patient had been subject to severe headaches since the age of eight and in half of these she would say everything is going black, and for 15 to 20 minutes she complained of not being able to see. Thereafter, a headache appeared. The only family history of headache was recurrent sinus pain in her mother. The patient's boyfriend said she had gone blind once before in his presence and that was after eating spaghetti.

Comment: Once more the events - blindness, weakness, and confusion - were consistent with embolism to the distal basilar artery. Blindness or inability to see had preceded her ordinary headaches. Tremor and ataxia developed with a delay of a month just as occurred in other cases. This is compatible with involvement of the thalamus or midbrain rather than the cerebellar hemisphere in which the deficit appears immediately.

Case 5: A woman aged 23 while seated after supper at 1800 , was suddenly struck with a severe pain in the left suboccipital region and immediately developed a pins and needles feeling throughout the entire right side - face, arm and leg. She cried out, fell forward over the table and began to vomit. She looked at the clock and noted that something was wrong or missing in the right upper visual field. On examination in the hospital 30 minutes later, she could only weakly move the right limbs and the right side of the face was weak. She felt "in a fog". She soon improved and after four days could walk unaided. The initial stab of pain lasted only a few seconds and was followed by a bad headache for $1 \frac{1}{2}$ days. Examination two weeks from the onset disclosed a right upper quadrant visual field defect, and slight weakness and slight impairment of sensation on the right side. The right pupil was $5 \mathrm{~mm}$ in diameter, the left pupil was $4 \mathrm{~mm}$. The ocular movements were full without nystagmus. The patient complained of a waving rolling visual impression in the right upper visual field. There was no constructional apraxia and memory was spared. The blood pressure was $95 / 65 \mathrm{~mm} \mathrm{Hg}$.

Tests for systemic disease were negative. The CSF was normal. A technetium brain scan and left carotid angiogram were normal. An electrocardiogram and electroencephalogram were unremarkable.

For several years the patient had been subject to recurrent weekly frontal throbbing headaches requiring $0.6 \mathrm{~g}$. of aspirin. They often occurred on arising in the morning. There had never been visual or paresthetic accompaniments. The patient's mother and sister had migraine headaches. The patient was not taking oral contraceptives. She smoked 30 cigarettes daily.

Examination nine months from the onset showed the right visual field defect and slight weakness of the right limbs which still felt numb. She used the left hand for eating because of a tremulousness of the right hand worse in action. The right foot made involuntary tapping movements. Five years later there was a constant wide amplitude tremor of her right hand not worse on intention.

Comment: This case has much in common with several of the other cases. It is another example of the delayed onset of tremor. Awareness of the field defect at the onset and the 
presence of rolling wavy vision place the lesion in the territory of the PCA. Angiography did not include the left PCA.

Case 6: (This case was included as Case 43 in a previous paper: Fisher 1971.) A woman aged 42, one morning, experienced a severe pain deep behind the medial part of the right eyeball. It lasted two seconds. Within a few seconds the left hand tingled and within three or four seconds the whole limb became numb. Her eyes felt crossed as if pulled down and in. She walked to her bedroom and lay down, called for help and in a few minutes, when helped to her feet, her legs were weak and did not support her. The right eyelid drooped and when the lid was lifted, she experienced vertical diplopia. There had been no prodromal symptoms and the patient did not lose consciousness at the onset. Examination at this time showed moderate paresis of the face, arm and leg on the left side, impaired sensation on that side and a right third nerve palsy. There was no visual field defect. The diplopia disappeared in two days and the ptosis in two weeks, but difficulty in focussing persisted. Within two months the patient walked with a spastic limp, whereas the left arm was literally normal and could be used for drying dishes, lighting a match, etc. The sensory impairment receded until there remained numbness of only the fingertips and corner of the mouth on the left side.

Two and a half months from the beginning suddenly one day, while combing her hair, she noted the onset of a tremor of the left arm such that her hand beat in front of her face. Concomitant with the tremor function in the left arm began to deteriorate slowly and gradually and in two or three months became spastic, useless and weak. When she walked, the left arm tended to extend behind her back. Walking became more difficult.

In the acute stage of her illness complete angiography was normal, as was the CSF. A pneumoencephalogram was within normal limits. No source of embolism was discovered. The blood pressure was 120/70.

On examination eight months from the onset of her illness the patient's mind was clear. There was a marked left facial weakness. When the left arm was held in forward flexion, there was a complete wrist-drop. Flexion and extension at the elbow were $7 / 10$ in power. Hand grip and abduction and adduction of the fingers were $5 / 10$. There was a marked limp and a pronounced foot-drop.

A $3 / \mathrm{sec}$. tremor involved the left shoulder and pronation-supination of the left forearm. The amplitude of the rhythmic elevation of the shoulder was $1 / 4$. The forearm rotation moved the little finger through a range of $3 / \mathrm{A}^{\prime \prime}$. The tremor was not accentuated on the finger-nose test. According to the patient, the left leg trembled at times, but the face and neck were not involved.

Eye movements were full without nystagmus. Sensation for pinprick and light touch was questionably reduced on the left side of the face.

The patient had had headaches all her life "just like everybody". There had never been accompaniments. The patient's mother and sister had migraine headaches. The patient had been receiving injections of an estrogen preparation twice a month for several years because of metrorrhagia. Her headaches had not become worse with this medication.

Comment: There can be no doubt that the territory of the right PCA was involved (right third nerve palsy and left sided weakness and numbness). The clinical events were compatible with embolism, but no source was found. This case illustrates the delayed onset of a resting tremor in the involved limbs this time associated with gradually decreasing function in the parts.

Case 7: A woman aged 29 taking oral contraceptive medication suddenly went blind at 8:30 p.m. "I couldn't see anything, vision went dark." In the same instant, the left side of the face and the left hand, fingers, and arm tingled. This lasted one minute. The line of demarcation passed down the middle of the nose and the left half of the lips felt swollen, as if novocainized. Fifteen minutes from the onset she developed a "bad" headache at both temples, worse on the right side, at least it throbbed on that side as she held her head in her hands. The headache was subsiding after two hours, but for several days afterwards, on shaking her head, she felt a "bruised" pain inside the skull. About three hours from the onset a small window of vision returned estimated as $2.5 \mathrm{~cm}$ wide at a distance of two meters. She could see her doctor's left eye and half of his moustache. By the next day $60 \%$ of the right visual field was restored, but none of the left. There was a small area of grayness near the central meridian in which there was the constant appearance of rolling waves. Five days from the onset neurological examination was normal except for a dense left homonymous hemianopia. A CT scan performed two hours from the onset was normal. A later CT scan showed a large low density area in the right PCA territory.

The patient was subject to headaches once or twice a year after the age of 15 . They were at one or the other temple, were throbbing in character and at times caused her to miss a day's work. There had never been any visual or paresthetic accompaniments. Her father, mother and two sisters had recurrent headaches. The patient had been taking estrogenic oral contraceptives for seven years. The usual periodic interruption had occurred seven days before the visual episode and had been followed by a normal menstrual period the last day of which coincided with the day of the spell. The patient's reading became normal; she returned to work, and was able to play tennis.

Seventeen months later, while she was lying in bed at 7:30 a.m. and having been awake for half an hour, suddenly vision narrowed until there remained only a narrow slit of central vision. "My vision's gone again." She could see only the tops of things. Looking in a mirror, she saw the lower half of her eyes and the face down to the chin, but she did not see her hair. The preserved field of vision gradually extended downwards, as well as widening out to the right. After several hours, she could see another person from forehead to waist and the left shoulder was visible. Some extension of the field continued and 18 days from the onset she could see down to the level of a sink. There was some sensitivity to light in the right visual field and in the upper central field there was again the impression of swirling clouds rolling in. There had not been a headache, but for several days shaking the head produced a bruised feeling at the temples "as if my brain was hitting against the skull."

Examination two weeks after the onset showed an island of vision in the right inferior quadrant of each eye abutting on the vertical meridian and extending about 30 degrees downwards and laterally. Otherwise, the examination was normal. Visual acuity was good and she could see to get around in familiar surroundings. Memory and intellect were intact. In the period between the two spells, she had had an occasional slight headache. The second visual episode occurred one or two days before her next menstrual period, which did not appear and it turned out that the patient was pregnant.

A CT scan five days after the onset showed the old infarct in the right occipital lobe and in addition a new low density area in the left occipital lobe with gray matter enhancement. Digital subtraction angiography was normal.

Echocardiography did not show mitral valve prolapse Systemic disease was ruled out.

Comment: This case illustrates sudden successive permanent insults to each occipital lobe without a history of classical migraine and associated in the first episode with oral contraceptive and in the second with early pregnancy. Headache, although definite, was not prominent and paresthesias accompanied the first episode.

Case 8: A healthy normotensive woman aged 51, while standing in her kitchen, suddenly felt slightly dizzy and faint and immediately developed a blinding generalized throbbing headache. She had to be supported as she became nauseated and began to vomit. Almost from the beginning she was aware of waviness of vision and inability to focus. The headache was severe for four days, but had subsided by the seventh day. Ability to focus improved, but a moving blurry waviness continued in the left visual field and she was found to have a left homonymous hemianopia. Neurological examination on day 10 showed the left field defect limited to the upper quadrant. The examination was otherwise normal.

A CT scan on day 10 demonstrated a large low density area in the right PCA territory associated with gray matter enhancement. Examination of the CSF on day 10 was normal. Angiography showed no abnormality of the PCAs or basilar artery. Echocardiography revealed prolapse of the posterior mitral leaflet. An extensive laboratory investigation was otherwise normal. 
In the following weeks "moving wavy lines" continued in the left visual field. The patient was subject to recurrent steady headaches occurring two or three times a month. About 15 years before on a few occasions she had an episode of scintillation and wavy lines lasting about 20 minutes and diagnosed as ocular migraine.

Comment: The development of a visual field defect was accompanied by the sudden onset of severe headache. Subarachnoid hemorrhage was probably.excluded. A cardiogenic embolus to the right PCA was a possibility, but severe headache is unusual in embolism. There has been no recurrence in four years.

Case 9: A woman aged 28 took her first oral contraceptive pill in six months at 9:30 a.m. one day. At noon she suddenly felt faint, was unable to focus her eyes and noted ringing in her right ear. Immediately she was struck by a severe pain in the left forehead and developed numbness of the right side of the face and the right arm and leg. There probably was some weakness of the right side, but the sensory loss was severe and weakness may have only been apparent. Neurological examination later in the day disclosed a right homonymous hemianopia, slight nominal aphasia, impaired memory, confusion and a severe deficit throughout the right side to sensation for pin, touch, vibration and joint position. A technetium brain scan showed an abnormal uptake in the left occipital region. Angiography was not performed. Investigation for an embolic source, arteritis, lupus erythematosus, etc. was negative. The right visual field defect cleared, but the patient was left with a persistent sensory deficit which evolved into a severe thalamic pain syndrome, which was accompanied by depression and the wish to die. There was a very active choreoathetosis at the fingers and wrist on the right side. There was a slight cerebellar ataxia of the right leg, but not of the arm. The patient had been subject to a headache twice a year since the age of 18 .

Comment: Angiography and a CT scan would have added greatly to this case. The clinical picture is classical for infarction in the PCA territory and inconsistent with hemorrhage. Embolism from an undetermined source cannot be excluded. Whether the event bore any relationship to the single oral contraceptive pill can only be decided in the light of greater experience.

Case 10: A woman aged 38, while falling asleep one night, suddenly experienced the most terrific, terrible pain like an explosion at the top of her head, but deep in. Immediately, her entire left side felt numb and the left arm and left leg were simply missing. On moving the left arm with the right, it felt like a piece of rubber. She fell asleep and the numbness was present the next morning. Two months before the patient had awakened one night with similar, but a less severe numbness of the entire side lasting ten minutes. After three or four days, the left leg no longer was numb, but there had developed a terrible burning sensation throughout the left side - face, arm, and leg. Contact with anything caused a burning sensation. This hypersensitivity gradually abated in the following weeks and the patient was left with a chronic thalamic pain syndrome involving all the fingers and the face. There was an involuntary movement in which the left shoulder elevated and the head tilted to the left. The left arm developed a semi-rhythmic shaking not worse on intention.

When examined two years later she reported she had had only three weeks free of pain in that period. She was tormented by a feeling of frost bite in the face, a squeezing sensation in the arm and aching in the fingers. Pinprick and light touch were slightly reduced throughout the left side - face, arm and leg. Pinprick produced hyperalgesia. Joint position sense, vibration and stereognosis were all reduced. There was choreo-myoclonic activity in the left forearm and fingers.

Two complete angiographic studies each showed an irregular narrowing of the right posterior cerebral artery in the region of the origin of the anterior temporal branch. A CT scan showed a questionable low density focus in the right lateral thalamus.

The patient had had only occasional headaches in the past, not requiring analgesics. She had had such a headache the day of the stroke. The terrible pain at the onset lasted only a few seconds. Her mother and two brothers were subject to migraine headaches. She had not used oral contraceptives for two years. She had had several years of psychotherapy attempting to gain equanimity in her marriage.

Comment: This case exemplifies the catastrophic onset of the Dejérine-Roussy syndrome in a normotensive young woman whose posterior cerebral artery showed minor stenosis.

\section{Summary of the Case Histories}

There were nine females and one male. Their ages ranged from 18 to 51 with seven under 35 years. In nine of the ten patients headache was prominent at the onset and six reported being dramatically stricken with a severe, sharp localized pain in the forehead or occiput. Visual symptoms were prominent at the onset in seven patients - four experiencing blindness and three a hemianopic deficit. Hemisensory symptoms or deficit occurred in six instances, a hemiparesis in three, combined weakness and sensory deficit in one and the limbs were spared in one (Case I had two episodes). Evidence of a third nerve palsy was found in three cases. A persisting neurologic deficit occurred in ten cases - visual field defect - six cases, hemiplegia - one, slight weakness - one, and a sensory deficit - two. A movement disorder developed on the involved side in seven cases. Evidence of infarction in one or both occipital lobes was obtained in six patients. One patient did not have impaired visual fields and the other three were examined before the days of nuclear medicine and CT scanning. Conventional angiography was performed in eight patients with the following results: retrothalamic occlusion of one PCA (one patient), distal occlusion of one PCA (one patient), retrothalamic narrowing of one PCA (one patient), irregularity of the wall of the upper basilar artery and both PCAs (one patient), and in four angiography was normal. A digital subtraction angiogram in one patient was normal and one patient did not have an arteriogram. A history of accompanied migraine was obtained in three patients. One patient was pregnant and one was three months post-partum. One patient was taking oral contraceptive pills, one patient had taken one contraceptive pill and one patient was receiving injections of estrogen.

\section{Discussion}

Cases 4 and 6 were included in a previous paper on unfamiliar types of cerebral ischemia. ${ }^{1}$ It was not appreciated at that time that Infeld ${ }^{2}$ had described a case in 1901 which was remarkably similar to the present cases and in which the process was deemed to be migrainous. His patient was a woman, age 28 , with a history of migraine who, during a usual headache, suddenly experienced tingling of the left side of the head, a warm feeling on the right side of the face above the ear and the sensation of a lightning flash through the right side of the body. She collapsed with paralysis and loss of sensation in the right side, including the face, arm and leg. The right hand tingled. The mouth deviated to the left. There was a dazzling appearance in the right eye. Diplopia did not occur. In the hospital she was mentally clear, the right side of the face was normal, the right arm and leg were slightly weak, sensation was intact and the tendon reflexes were normal. Full recovery occurred, but one month later "spasms" began in the right arm. Examination seventeen months later showed a slight right sided weakness with slightly increased reflexes. Sensation was normal, but the right thumb and index finger tingled. There were adventitious movements consisting of rather rapid pronation-supination of 
the hand and flexion-extension movements of the fingers, which the author interpreted as lying between athetosis and chorea. The movements were not regular and were exaggerated on the finger-nose test. In this case, the association of the attack with one of the patient's ordinary migraine headaches offered good evidence in favor of migraine as the underlying process. This matter will be discussed more fully later.

The Localization of the Process. The clinical details of each case, especially when combined in some of the cases with the findings on CT and angiography, provide convincing evidence that the territory of one or both PCAs was involved. On each side the PCA supplies the third nerve complex, the cerebral peduncle, the superior projection of the cerebellar outflow, the sensory nucleus of the thalamus and the visual radiations and cortex of the temporo-occipital lobe. On the dominant side involvement of the hippocampus and inferior temporal lobe result in impaired memory, confusion and nominal aphasia. The locus of action along the PCA must have been close to the origin of the artery in those cases with the third nerve palsy and/or hemiparesis (Cases 1 to 6). In the other cases, only the more distal territory, sensory and visual, may have been involved. In no case were there symptoms that clearly lay outside the PCA territory. In Case 10 only the sensory system, presumably in the thalamus, was involved, but in that case the PCA on the appropriate side was stenotic.

The Nature of the Process. The abrupt, almost catastrophic development of the clinical picture immediately suggests a cerebrovascular process. Since there have been no pathological studies, the best one can do is to consider to what extent each of the known kinds of vascular processes fits the clinical and laboratory findings.

The sudden onset without prodromal TIAs makes cerebral embolism a prime suspect. But in no case was there an obvious embolic source. It is possible that mural thrombus lying more proximally in the vertebral-basilar system was dislodged, but angiography failed to reveal any such thrombus. In three cases there was bilateral PCA involvement without loss of consciousness or abulia which would be expected when an embolus is arrested at the distal end of the basilar artery. In Cases 1 and 2 the appropriate PCA was the site of an obstruction consistent with an embolic plug which had not undergone lysis. In Cases 3 and 4 the arterial abnormality was not in keeping with embolism and in the remainder of the cases there was no obstruction. In Case 1 the symptoms were on the left side the first time and on the right side the second time requiring that successive emboli from an unknown source entered opposite PCAs, a most unlikely event. Does known embolism to the PCA produce the striking headache experienced by most of these patients? A review of 25 personal cases of reasonably certain PCA embolism, mainly in association with atrial fibrillation, showed that seven had a headache and in only five was it present at or near the time of the onset of the visual field defect. In only two was the headache a prominent complaint and in only one was it the dominating feature, as in the present cases. In summary, the evidence against embolism is strong on several points, but total exclusion of the possibility can be made only with reservation.

Thrombosis of the PCA is not a likely candidate since in none of the cases did angiography show obstruction at the appropriate site to account for the patients' symptoms. An obstructing thrombus-in-situ does not undergo lysis as does embolic material.
The blockage of vessels seen angiographically was so situated as not to account for the patients' symptoms.

The possibility that a migraine-like process was operative must be a serious consideration. Infeld's case (1901) has already been mentioned. The present patients were generally young. Severe headache was a consistent symptom. The visual system of the PCA territory, and presumably the PCA itself, normally are fundamental participants in migraine accompaniments, for $90 \%$ of all migraine accompaniments involve the visual system alone or along with accompaniments of another modality such as paresthesias, aphasia, etc. Transient blindness, either total or hemianopic, is by no means rare as a migraine aura. A persistent hemianopia is a widely recognized sequela of ophthalmic migraine. According to Davis-Jones et al., ${ }^{3}$ at least 145 cases of persisting neurologic deficits after migraine have been described in the literature. Many of these have consisted of a hemianopia, for example, six of the 18 patients of Connor, ${ }^{4}$ and eight of nine patients of Davis-Jones et al. ${ }^{3}$ A personal experience with 21 such cases was previously referred to (Fisher 1971) and the number has continued to increase. It is, of course, impossible to prove that the hemianopia in these cases is migrainous, but in the absence of an alternative etiology, it is the only plausible interpretation. Although only three of them had a history of accompanied migraine, if one is obliged to assign the present cases to a known pathophysiologic mechanism, it is our view that migraine is the most likely process. The nature of the action of migraine on arteries remains unknown, but whatever it is, the present cases exemplify the process in an especially severe form.

If the cases are migrainous, they are good evidence that paresthesias, sensory loss, weakness and diplopia may arise, at least at times, in PCA territory rather than middle cerebral or basilar artery territory.

In Case 7 the patient was taking oral contraceptive medication at the time of the first attack and was in the early stages of pregnancy in the second spell. In patients receiving oral contraceptive medication headaches may develop for the first time, migraine headaches may become more frequent or more severe and a persistent hemianopia may be precipitated. Is there a link in the present cases with the cerebrovascular complications of oral contraception? For example, a woman aged 34 receiving oral contraceptive medication suddenly became totally blind while taking a shower bath and at the same time developed a severe throbbing occipito-nuchal pain. Although uncertain, she estimated that the blindness lasted several minutes. The headache subsided in two weeks. Detailed studies revealed no underlying disease and a diagnosis of migraine was made. In the light of this case a review of the literature on the subject of oral contraceptives revealed no other such case and none resembling the ten described herein. Nevertheless, there is some analogy between the present cases and the process at work in the cerebrovascular complications of oral contraceptives. But the nature of that process is also obscure.

The Movement Disorders of Delayed Onset. Movement disorders not particularly well characterized were surprisingly common in these cases, although the patients were in no way selected on that basis. Movement disorders developed in seven of the cases and involved the side most affected by the initial stroke. The interval between the stroke and the onset of adventitious movements was respectively: Case 1, uncertain, present 
at five weeks; Case 2, two months; Case 3, uncertain, present at seven weeks; Case 4, one month; Case 5, uncertain, present at nine months; Case 6, two-and-one-half months; and Case 8, uncertain, present at three years.

The movement disorders took the form of tremor, cerebellar ataxia or choreoathetosis. Case 1, slight cerebellar ataxia on the finger-nose test, gross dysmetria on finger tapping; Case 2, resting tremor and choreic activity of the left hand; Case 3, slight cerebellar dysmetria in the left arm and action tremor in the left fingers; Case 4 , rhythmic 3/sec. tremor of fingers and arm on extending the arm, no tremor at rest; Case 5, a constant wide-amplitude tremor of the right hand, not worse on intention and involuntary tapping movements of the foot; Case 6, 3/sec. tremor involving the left shoulder and pronation-supination of the left forearm not accentuated on the finger-nose test, trembling of the left leg at times; Case 9, choreoathetosis of the right hand and slight cerebellar ataxia of the right leg.

In summary, a resting tremor was present in four cases, cerebellar ataxia in two and choreoathetosis in one. Hemiballismus was not reported in the acute stage in any case. The choreoathetosis was associated with a severe thalamic sensory deficit. The cerebellar ataxia is traceable to involvement of the superior cerebellar outflow after decussation, either in midbrain or thalamus. Tremor is less easy to account for and a clearer description of it would be desirable before speculating. The patients who developed a tremor had, in the acute phase, sustained a hemiplegia due to involvement of the peduncle or internal capsule. If it was the peduncle, a lesion of the substantia nigra or its upward projections might be postulated. In cases in which the tremor was accentuated by action, it may be related to the superior cerebellar outflow, so called "rubral" tremor. The basis of the delayed onset of the tremor is of considerable interest and in terms of modern knowledge of neurotransmitter physiology suggests a gradual loss of synaptic transmitter following deafferentation.

Do the cases constitute a syndrome? Do these cases have enough in common to warrant the term "clinical syndrome" ? Certainly they represent involvement of the territory of the PCA. They share the same features in varied combinations. The onset or evolution is dramatic, distinctive and even alarming. The cases do not easily fall into any commonly recognized category of cerebrovascular disturbance. As already discussed, the process that most likely applies to this group of cases is migraine. If that is so, the term "catastrophic migraine" or "cataclysmic migraine" may have some currency.

Concerning therapy. Without knowledge of the underlying process and since cerebral damage will have been established when the patient first comes to medical attention, it is difficult to conceive of effective measures. If it is assumed that the process is ischemic and since vascular obstruction was found in two cases, the possibility of using heparin therapy might be considered. In most of the present cases, steroid therapy was used to control brain swelling. If the pathologic process is temporary vasospasm, the use of hemodilution or hyperbaric oxygen could be an option.

Two patients (Cases 1 and 7) experienced two sudden episodes and in this regard it may be mentioned that in our experience permanent sequelae in migraine are recruited from patients whose accompaniments come on suddenly without gradual "build-up". It is in such patients that a trial of long term therapy with Coumadin is undertaken when migraine spells are increasingly frequent or when a limited permanent sequela has occurred.

\section{ACKNOWLEDGEMENTS}

This work was supported in part by a grant from the Freed Foundation, Washington, D.C.

I am grateful to Dr. Allan Ropper for allowing me to include Case 1.

\section{REFERENCES}

1. Fisher CM. Cerebral ischemia - less familiar types. Clinical Neurosurgery 1971; 18:267-336.

2. Infeld M. Zur Kenntniss der bleibenden Folgen des migräne Anfalles. Wien. klin. Wschr. 1901; 14:673-675.

3. Davis-Jones A, Gregory MC and Whitty CWM. Permanent sequelae in the migraine attack. 5th Migraine Symposium Heinemann, London 1973, 25-27.

4. Connor RCR. Complicated migraine. A study of permanent neurological and visual defects caused by migraine. Lancet 1962;2:1072-1075. 